\title{
Advanced Imaging Modalities to Monitor for Cardiotoxicity
}

Andreas Seraphim, $M B B S^{1,2}$

Mark Westwood, MBBS, PhD ${ }^{1,3}$

Anish N. Bhuva, MBBS $S^{1,2}$

Tom Crake, MBBS, PhD ${ }^{3}$

James C. Moon, $M D^{1,2}$

Leon J. Menezes, FRCR FRCP ${ }^{1}$

Guy Lloyd, MD ${ }^{1}$

Arjun K. Ghosh, MBBS, PhD ${ }^{1,3}$

Sarah Slater, $M D^{4}$

Heather Oakervee, $M D^{5}$

Charlotte H. Manisty, MBBS, PhD ${ }^{1,2,3 \text {, * }}$

\footnotetext{
Address

${ }^{1}$ Department of Cardiovascular Imaging, Barts Heart Centre, Barts Health NHS Trust, West Smithfield, London, EC1A 7BE, UK

${ }^{2}$ Institute of Cardiovascular Sciences, University College London, Chenies Mews, London, UK

*,3 Department of Cardio-oncology, Barts Heart Centre, Barts Health NHS Trust, West Smithfield, London, EC1A 7BE, UK

Email: c.manisty@ucl.ac.uk

${ }^{4}$ Department of Haematology, Barts Health NHS Trust, West Smithfield, London, EC1A 7BE, UK

${ }^{5}$ Department of Oncology, Barts Health NHS Trust, West Smithfield, London, EC1A 7BE, UK
}

Published online: 8 August 2019

(C) The Author(s) 2019

\section{This article is part of the Topical Collection on Cardio-oncology}

Keywords Cardiotoxicity · Cardiac imaging • Chemotherapy · Cancer treatment · Cancer · Cardiac MRI ·

Echocardiography

\section{Opinion statement}

Early detection and treatment of cardiotoxicity from cancer therapies is key to preventing a rise in adverse cardiovascular outcomes in cancer patients. Overdiagnosis of cardiotoxicity in this context is however equally hazardous, leading to patients receiving suboptimal cancer treatment, thereby impacting cancer outcomes. Accurate screening therefore depends on the widespread availability of sensitive and reproducible biomarkers of cardiotoxicity, which can clearly 
discriminate early disease. Blood biomarkers are limited in cardiovascular disease and clinicians generally still use generic screening with ejection fraction, based on historical local expertise and resources. Recently, however, there has been growing recognition that simple measurement of left ventricular ejection fraction using $2 \mathrm{D}$ echocardiography may not be optimal for screening: diagnostic accuracy, reproducibility and feasibility are limited. Modern cancer therapies affect many myocardial pathways: inflammatory, fibrotic, metabolic, vascular and myocyte function, meaning that multiple biomarkers may be needed to track myocardial cardiotoxicity. Advanced imaging modalities including cardiovascular magnetic resonance (CMR), computed tomography (CT) and positron emission tomography (PET) add improved sensitivity and insights into the underlying pathophysiology, as well as the ability to screen for other cardiotoxicities including coronary artery, valve and pericardial diseases resulting from cancer treatment. Delivering screening for cardiotoxicity using advanced imaging modalities will however require a significant change in current clinical pathways, with incorporation of machine learning algorithms into imaging analysis fundamental to improving efficiency and precision. In the future, we should aspire to personalized rather than generic screening, based on a patient's individual risk factors and the pathophysiological mechanisms of the cancer treatment they are receiving. We should aspire that progress in cardiooncology is able to track progress in oncology, and to ensure that the current 'one size fits all' approach to screening be obsolete in the very near future.

\section{Introduction}

With more than 14 million new diagnoses of cancer in 2018 alone [1] and with half of patients expected to live for 10 years or more, the importance of healthy survivorship in oncology is increasingly important. Improved survival rates alongside the wealth of novel therapies prescribed to older patients with more co-morbidities have resulted in greater incidence of cardiac complications during cancer treatments, which then negatively impact cancer outcomes. Early detection and treatment of emergent cardiotoxicity has been shown to both reduce cardiovascular adverse events [2], and enable better treatment of the underlying cancer. However, overcautious diagnosis and management from a cardiac perspective in this context may prevent patients from receiving optimal cancer treatment, thereby impacting on remission and survival rates.

Cardiooncology is a rapidly developing subspecialty within cardiology which aims to optimize diagnosis and management of cardiac complications of cancer treatment $[3,4]$. Unfortunately our understanding of the underlying pathophysiology and natural history of cardiotoxicity remains limited, and it is generally only detected once cardiovascular disease presents clinically $[5,6]$. Over the past decade, the armoury of anti-cancer therapies has expanded enormously [7], many of which are targeted therapies based on tumour genetic and receptor profiles, rather than simply tumour location. Unfortunately these are accompanied by a growing range of cardiovascular sequelae, including not only heart failure and left ventricular systolic dysfunction (cancer therapeuticsrelated cardiac dysfunction, CTRCD) but also myocarditis, arrhythmias, thrombotic events, coronary, pericardial and valvular heart disease. Recognition of these cardiovascular effects has led to screening being incorporated into both clinical pathways and research trials. However, how this is best achieved and with which imaging biomarkers and modalities remains controversial. Cardiac imaging has progressed significantly over the past two decades, and advanced echocardiographic techniques including three dimensional imaging, cardiovascular magnetic resonance (CMR) imaging, computed tomography (CT) and nuclear techniques including positron emission tomography (PET) have the potential to improve diagnostic accuracy of screening and offer 
an insight into the underlying pathophysiology of cardiotoxicity.

The decision to employ one imaging modality over another depends on several factors, including the technical and professional resources available, financial costs, patient preferences and modality-specific advantages and limitations (Table 1). An ideal imaging biomarker should be accurate and precise (with excellent inter-study reproducibility for serial screening), give an insight into the underlying pathophysiology and have sufficient sensitivity to detect disease before it becomes clinically apparent. This has been recognized in the context of cardiotoxicity for many years [8], but now more than ever the additional considerations of cost, availability and safety (lack of ionizing radiation) have governed decision making, rendering adoption of advanced imaging modalities in routine clinical workflows more challenging.

The focus of this review is to give an overview on the use of advanced imaging modalities for cardiotoxicity monitoring, focusing on their diagnostic capabilities and limitations as well as their potential future applications.

\section{Monitoring for cancer treatment-related cardiac dysfunction}

The cardiotoxic effects of anthracyclines and trastuzumab on cardiac function have long been recognized; however, many of the newer targeted therapies including tyrosine kinase inhibitors and immunotherapy are also associated with cardiac dysfunction. Baseline assessment of cardiac structure and function prior to initiating potentially cardiotoxic cancer treatments is essential, particularly in those at higher risk $[9 \bullet \bullet, 10 \bullet \bullet, 11 \bullet \bullet, 12 \bullet \bullet, 13 \bullet \bullet, 14]$. This is both an important component of initial risk assessment prior to start treatment, but also avoids inappropriately ascribing abnormalities detected during treatment to the therapy, in patients with pre-existing cardiomyopathies.

Left ventricular ejection fraction (LVEF) is the principal marker of left ventricular systolic function currently used both in clinical practice and research, and early asymptomatic declines are associated with subsequent progression to clinical heart failure in the context of cancer treatment [15-17]. Serial imaging is therefore recommended before, during (for HER2-targeted treatments) and on completion of treatment with anthracycline or other cardiotoxic agents. Cancer therapeutics-related cardiac dysfunction (CTRCD) has been defined as a drop in LVEF $>10 \%$ to below the lower limits of normal, although different absolute

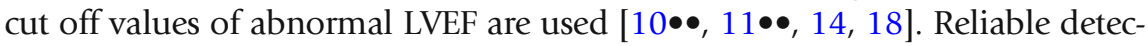
tion of CTRCD therefore depends not only on the sensitivity and accuracy of the imaging method to detect subtle changes in LVEF but also the ability to discriminate true changes in ejection fraction between studies from background noise (precision). This is determined by limitations in image quality and intraand inter-observer reproducibility [19].

Echocardiography is recommended first line for cardiotoxicity screening by

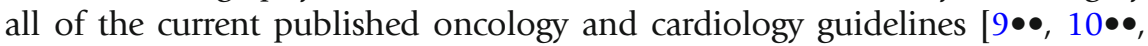
$11 \bullet \bullet, 12 \bullet \bullet, 13 \bullet \bullet, 14]$. There are inherent advantages to the technique: low-cost, widespread availability, lack of ionizing radiation and patient acceptability; however, the accuracy of 2D echocardiography is limited by its reliance on geometric assumptions and adequate acoustic windows (potentially worse in cancer patients, for example post-mastectomy). Test-retest variability in LVEF measurement by 2D echocardiography is however up to $10 \%[19,20]$ and it has been questioned whether it can reliably detect the 5-10\% change used to define 


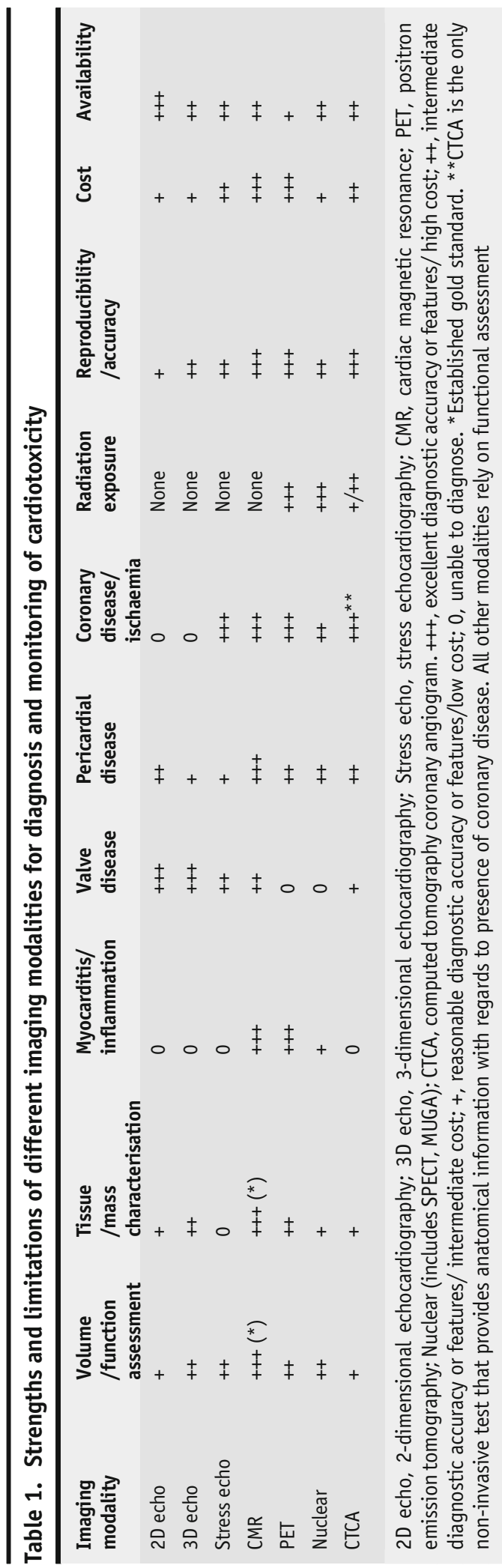


CTRCD [21]. The use of transpulmonary contrast results in a higher level of precision than 2D alone, especially when windows are limited [22] and forms part of international LV assessment guidelines [23]. 3D echocardiography is a more precise method for measurement of $\mathrm{LV}$ volume and function against a gold standard of CMR [24-27]. Unfortunately, however, it is feasible in only $60 \%$ of patients post-anthracycline chemotherapy for breast cancer [28], due to poor echocardiographic windows in this population

Multigated acquisition (MUGA) scans [29] have historically been widely used for LVEF evaluation, and were the imaging modality of choice in clinical trials of anthracycline cardiotoxicity in the 1980s due to widespread availability [15]. Despite good intra- and inter-observer reproducibility [30], measures of LVEF by MUGA are only modestly accurate when compared with a gold standard of CMR, with misclassification of $35 \%$ of subjects when cardiotoxicity was diagnosed using a LVEF threshold of 50\% [31]. MUGA is further limited by both the associated radiation exposure, and the limited information it provides on other cardiac structures. Single-photon emission computed tomography (SPECT) enables acquisition of 3D images and provides an additional option for LVEF evaluation. It can provide information on right ventricle function and wall motion abnormalities; however, it tends to underestimate LVEF values compared to MUGA and echocardiography [32]. Positron emission tomography (PET) is unlikely to have a widespread role in screening for cardiotoxicity despite its accuracy [33], because of high cost and radiation exposure along with limited availability.

CMR imaging is now the gold standard for evaluation of ventricular volumes and function [34], with proven superior reproducibility for LVEF assessment [35]. LVEF is calculated from a stack of short axis cine images of the heart, with the endocardial borders segmented either manually or automatically at end-diastole and end-systole, in order to provide the cavity areas for each slice. Summation of the slice areas enables calculation of the LV end-diastolic and end-systolic volume, from which ejection fraction can be calculated. With temporal variability in LVEF measurements estimated at 2.4 to $7.3 \%$ [20, 36] and without the constraints of reliance on acoustic windows, CMR is wellsuited for monitoring for cardiotoxicity, particularly in those whose echocardiographic images are suboptimal [37]. The enhanced reproducibility of CMR over echocardiography for detecting small changes in LVEF translates into the potential for smaller sample sizes in clinical studies. CMR-derived measures of LVEF are now commonly used as the endpoint in randomized trials evaluating the value of cardioprotective agents for prevention of cardiotoxicity [38, 39]. Importantly, CMR also provides additional information on LV structure parameters such as LV mass, which has been shown to independently predict cardiovascular events in patients following anthracycline therapy [40]. Although both access to and costs of CMR have historically limited its use, rapid CMR protocols [41], 10 or $20 \mathrm{~min}$, can be adapted to cardiooncology, enabling cheaper, shorter scans that can deliver improved efficiency.

\section{Imaging biomarkers of early subclinical CTRCD}

Management of heart failure secondary to cancer therapies can be challenging if diagnosed late, with prognosis historically worse with anthracycline-related 
cardiomyopathy than other aetiologies of heart failure [42]. By the time a drop in LVEF is detected, the opportunity for maximal therapeutic intervention may have already been missed [43]. Data from 2625 patients followed during and after treatment with anthracyclines showed that the incidence of cardiotoxicity was $9 \%$, with $98 \%$ of cases arising within the first year following treatment, of whom the majority had at least partial recovery if treatment was started early [44]. Alongside this, histological data has found significant myocellular injury on biopsy despite preserved ejection fraction, suggesting that LVEF may be a relatively late marker of cardiotoxicity [45].

Although echocardiographic markers of diastolic function including tissue Doppler velocities have been explored in early cardiotoxicity, studies show conflicting results [46, 47] [48]. Stress imaging has also been explored as a potential tool for early detection of cardiotoxicity [49]. Stress echocardiography was shown to detect subclinical cardiac dysfunction in young adults treated with anthracyclines [50], but the incremental value of stress echocardiography and the role of contractile reserve in cardiotoxicity monitoring remains unclear [51, 52]. Myocardial deformation using left ventricular (LV) strain, strain rate and twist [53-55] by echocardiography are more sensitive and earlier biomarkers of cardiotoxicity than LVEF, and permit detection of cardiotoxicity at lower chemotherapy doses than were historically believed to be associated with cardiac damage [56]. A relative reduction of peak LV systolic global longitudinal strain (GLS) by 10 to $15 \%$ is an early predictor of subsequent cardiotoxicity [57-59], and 3D GLS may detect cardiotoxicity earlier than 2D GLS [60, 61]. The ongoing SUCCOUR trial [62] will be the first randomized controlled study using GLS as a predictive biomarker for CTRCD, and the results will likely impact clinical practice.

More recently, CMR-derived GLS (using feature tracking) has been shown to detect LV dysfunction before LVEF falls [63] and to be an independent predictor of all-cause mortality across all cardiomyopathies [64]. Reductions in both global circumferential and longitudinal strain have been demonstrated in patients receiving doxorubicin and trastuzumab, which correlated with changes in subclinical declines in LVEF [65-67], highlighting its potential use for monitoring of early cardiotoxicity from chemotherapy.

\section{Imaging biomarkers to understand the pathophysiology of CTRCD}

Left ventricular dysfunction is the most frequent final manifestation of cardiotoxicity, but may result from a variety of different, treatment-specific, pathophysiological mechanisms [68] including myocyte apoptosis [69], myocardial fibrosis, inflammation [70] and ischaemia [71]. An ideal imaging biomarker would interrogate individual pathways directly, to detect cardiotoxicity prior to the development of myocardial mechanical dysfunction. Furthermore, the introduction of new therapies targeting different treatment pathways, including novel immunomodulatory strategies such as adoptive T cell therapy (ACT) and immune checkpoint inhibitors (ICI), has resulted in new and less well-defined mechanisms of injury to the heart, often resulting in a wide spectrum of toxicity and clinical presentations, ranging from asymptomatic detection of elevated cardiac biomarkers to cardiogenic shock [72, 73]. Advanced imaging offers the potential for tissue characterisation, and hence the ability to detect myocardial oedema and inflammation, focal and diffuse 
fibrosis and assess myocardial perfusion.

CMR adds value because of its myocardial tissue characterisation capabilities with growing evidence within cardiooncology (Fig. 1). Gadolinium-based contrast agents can be administered to detect focal myocardial scarring and fibrosis, and the distribution and extent of myocardial scar can be used both to differentiate aetiologies of myocardial disease (for example ischaemic cardiomyopathy versus myocarditis) and to estimate prognosis [74, 75]. Although experimental models of anthracycline cardiotoxicity [76] demonstrated focal scarring on late gadolinium enhancement (LGE) imaging, clinical studies suggest LGE is rarely detected, and is not associated with outcomes $[77,78]$. Rather than focal scarring, anthracyclines are thought to cause diffuse interstitial fibrosis, via excess collagen deposition. Diffuse fibrosis can be quantified by CMR using
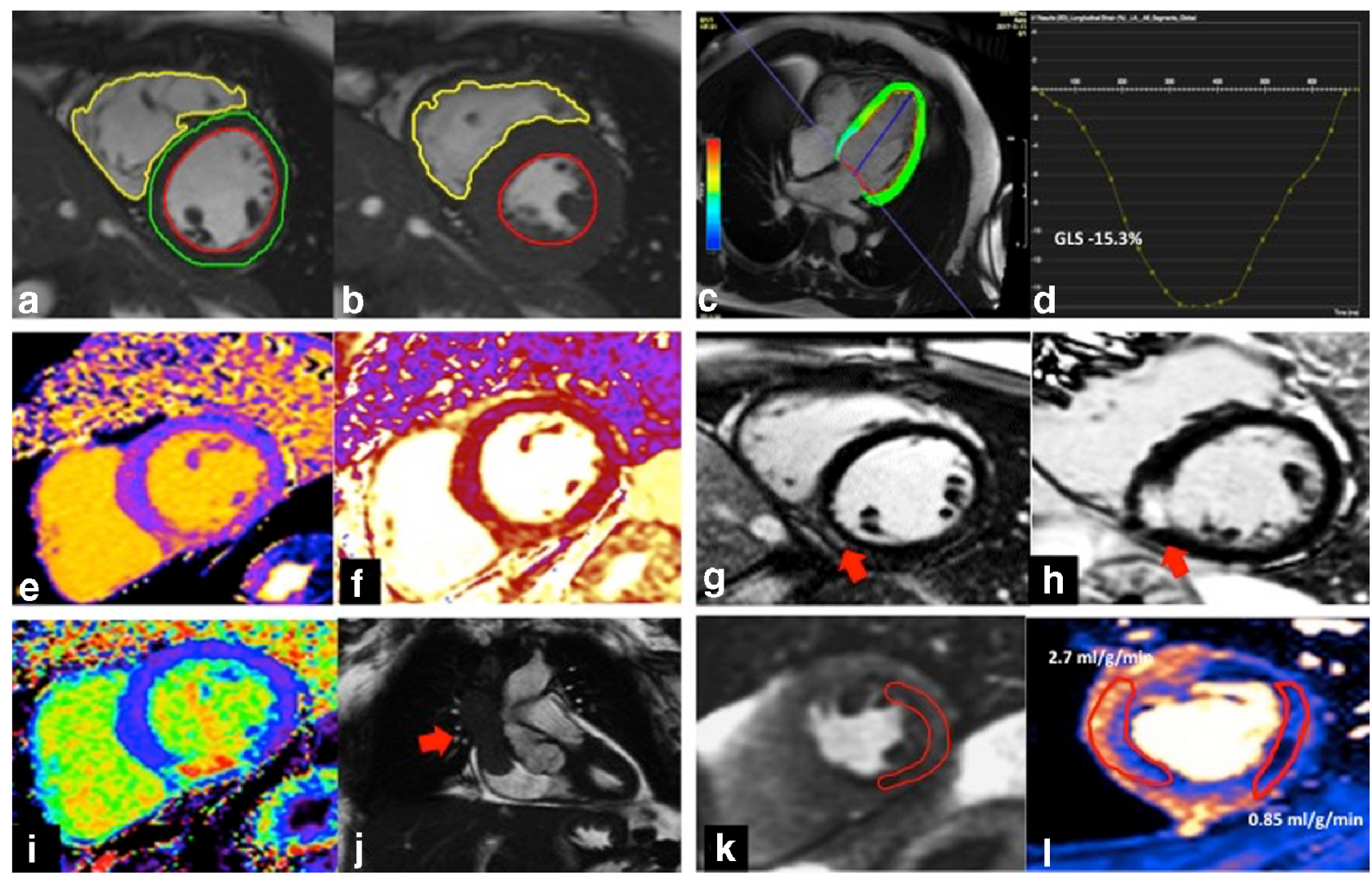

Fig. 1. Multiparametric cardiac evaluation using CMR. a-d Cardiac function and volumetric assessment: Endocardial and epicardial borders are drawn in diastole (a) and systole (b) allowing calculation of LVEF, chamber volumes and myocardial mass. Measurement of myocardial deformation using feature tracking CMR (c) enables measurement of global longitudinal strain (GLS), a marker of early, subclinical cardiotoxicity (d). e-j Tissue characterisation: Quantitative parametric mapping techniques such as T1 (e), T2 (f) and extracellular volume (ECV) mapping, (i) detect and measure diffuse myocardial fibrosis and oedema. Late gadolinium enhancement imaging $(\mathbf{g}, \mathbf{h})$ identifies focal fibrosis, and can differentiate between underling pathologies such as myocarditis (subepicardial, $\mathbf{g}$ ) and infarction (subendocardial, $\mathbf{h}$ ). Intracardiac masses ( $\mathbf{j}$, arrow), here in the superior vena cava and the right atrium, can be identified and tissue characterisation enables stratification of the underlying tissue type. $\mathbf{k}-\mathbf{l}$ Myocardial perfusion imaging can assess for functionally significant coronary artery disease and aid risk stratification pre-cancer treatment or surgery. Here, a region of relative hypo-perfusion (k, outlined myocardium) is seen with vasodilator stress, with reduced myocardial blood flow at $0.85 \mathrm{ml} / \mathrm{g} / \mathrm{min}$ demonstrated on the quantitative perfusion map (l), related to circumflex territory ischaemia. 
pre- and post-contrast T1 mapping, and this technique has been validated against biopsy-measured collagen volume fraction with good reproducibility across a spectrum of other cardiac disorders [79, 80]. T1 mapping has been explored as a biomarker of early anthracycline cardiotoxicity, with several small studies showing elevated myocardial T1 and extracellular volume fraction (ECV) in patients treated with anthracyclines compared with age- and sexmatched controls $[81,82]$. Other studies have however failed to reproduce these results $[78,83]$, particularly in lower risk patients. This conflicting data therefore means that, at least currently, T1 mapping is not a tool for anthracycline- or HER2-related mainstream cardiotoxicity screening.

CMR can also detect myocardial oedema and inflammation using T2weighted imaging sequences, with higher $\mathrm{T} 2$ relaxation times seen with increasing myocardial water content. Parametric mapping techniques have been developed to quantify $\mathrm{T} 2$ relaxation times, and recent data from a pig model of doxorubicininduced cardiotoxicity using serial multiparametric (T1, T2 and ECV) mapping has shown that $\mathrm{T} 2$ values provide the earliest marker of myocardial damage [84•]. Myocardial T2 values increased early following administration of anthracyclines, correlating with increase myocardial water content, despite normal T1 and ECV values and no detectable abnormalities in myocardial contractility. On stopping anthracycline administration when T2 levels increased, T2 values normalized and LV dysfunction did not progress, suggesting that cardiotoxicity may be largely reversible when detected at this early stage. Albeit from animal data with intra-coronary chemotherapy injection, this study provides evidence that $\mathrm{T} 2$ imaging may be a potential early imaging biomarker for cardiotoxicity with anthracyclines. T1 and T2 mapping techniques are also useful for the diagnosis and monitoring of myocarditis, which has been associated with various cancer agents, with increasing recognition in the context of immunotherapies [85]. Although there is insufficient data currently available to clarify the role of CMR in immunotherapy-related myocarditis, it is likely that the ability of this modality to detect both oedema and fibrosis will deliver clinical utility.

PET imaging can detect alterations in myocardial metabolism and inflammation [86], offering good sensitivity for the diagnosis of myocarditis and therefore potentially other cardiotoxicities. PET data [87] suggests that changes in myocardial glucose metabolism can be detected early in the course of anthracycline treatment, with low baseline myocardial ${ }^{18}$ F-FDG uptake predicting a progressive increase in cardiac glucose consumption during and after chemotherapy, as well as a higher incidence of cardiotoxicity [88].

\section{Evaluation of coronary artery disease and myocardial ischaemia}

Whilst cardiotoxicity related to cancer therapy generally focuses on left ventricular impairment [89], cancer treatments can cause other clinical cardiac syndromes including coronary events, pericardial disease, valvular heart disease, pulmonary hypertension and right ventricular dysfunction [90-94] (Table 2).

Several cancer treatments have been implicated in the development of myocardial ischaemia and coronary events including myocardial infarction, including fluoropyrimidines, platinum compounds, VEGF inhibitors, certain bcr-abl tyrosine kinase inhibitors and radiotherapy [10••] (Table 2). Pathophysiological mechanisms differ by drug and include accelerated atherosclerosis, coronary spasm, 


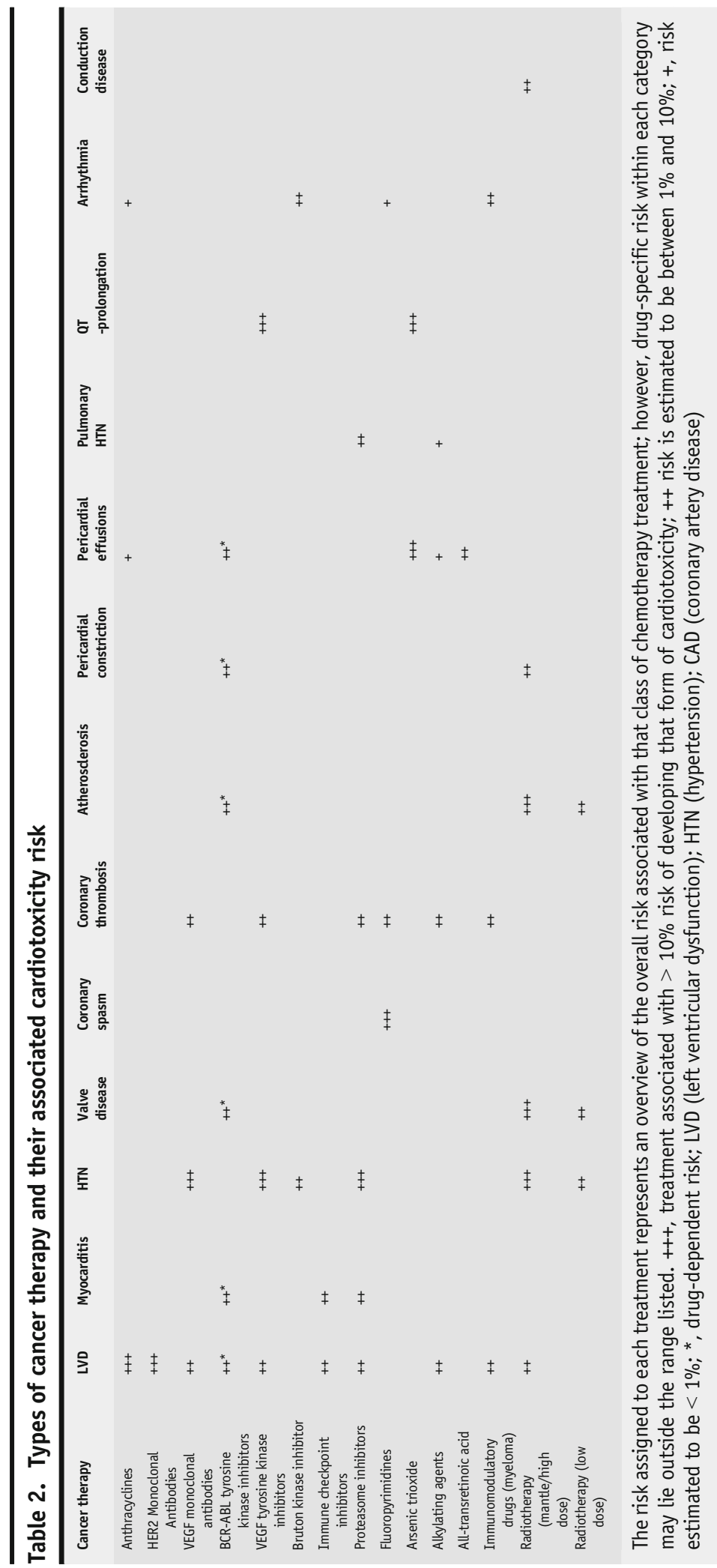


vascular endothelial damage and arterial thrombotic events. Most cardiac imaging modalities including echocardiography, CMR and nuclear imaging can be used for functional testing in this context, with exercise or stress agents including adenosine, regadenoson and dobutamine used to unmask ischaemia or myocardial perfusion abnormalities. There are small differences in the diagnostic performance of these tests [95]; however, the selection of an individual modality is generally based on local expertise and availability. CMR can sensitively detect myocardial infarction using LGE, and quantitative myocardial perfusion mapping offers the potential to directly quantify regional myocardial perfusion reserve [96], previously only feasible with nuclear imaging techniques. CT coronary angiography provides a noninvasive anatomical assessment of coronary artery disease, and with an excellent negative predictive value can offer a reliable test for exclusion of significant coronary disease and for risk stratification prior to surgery or administration of the drugs listed above.

\section{Valvular heart disease}

Valve disease is a rare complication of chemotherapy, however is wellrecognized as a late consequence of high-dose radiotherapy to the mediastinum (particularly with historical techniques such as mantle field radiotherapy). There is a latent interval of 10-20 years between radiation exposure and development of clinically significant heart valve disease, with risk related both to radiation dose and interval from exposure, with reported prevalence rates of $5-32 \%$ in patients treated for Hodgkins lymphoma [97]. Importantly, surgical outcomes in these patients are worse than in a matched cohort of patients undergoing valve replacement [98], meaning that early detection and accurate assessment is critical, with non-surgical, percutaneous valve implantation approaches playing an increasing role [99].

Echocardiography remains both the first-line and gold standard imaging modality for functional assessment of valvular heart disease, allowing qualitative and quantitative evaluation of both stenotic and regurgitant valves. Computed tomography and CMR [100] can also be used for valve evaluation, with the former being useful for stenotic valve planimetry and evaluation of suspected endocarditis [101], particularly when hybrid imaging such as PETCT is employed [102]. CMR can measure flow across valves using phase contrast imaging, and hence is often employed where echocardiographic assessments are of poor quality or uncertain [103].

\section{Pericardial disease}

Pericardial disease is a common finding with cancer therapy, and pericarditis, pericardial effusion and constrictive pericarditis are all seen associated with both chemotherapy agents (including anthracyclines, cytarabine, arsenic and tyrosine kinase inhibitors), and mediastinal radiotherapy [104]. Pericarditis can arise acutely during radiotherapy, leading to later pericardial constriction which typically presents over 10 years following treatment and has a cumulative incidence of up to $5 \%$ in this population $[10 \bullet \bullet]$. Echocardiography is the first-line imaging modality for pericardial assessment, including diagnosis and functional characterisation of 


\section{Pulmonary hypertension}

constrictive and tamponade physiology, whilst CT is able to reliably detect the pericardial calcification generally seen as a late complication of radiotherapy. CMR offers additional diagnostic information, combining both sensitive structural imaging (using dark blood T1 weighted imaging with and without fat saturation) with tissue characterisation (multiparametric mapping and LGE) and functional assessment (real-time cine imaging during free breathing) for more detailed investigation of pericardial disease [105]. This can be particularly useful where echocardiography is inconclusive, or where more detailed tissue characterisation is required. Formal diagnosis and evaluation of constrictive pericarditis can be challenging, and a clinical role remains for invasive cardiac catheterisation with haemodynamic assessment in some circumstances.

Although a rare complication of cancer therapy, development of pulmonary hypertension has been observed with dasanitib (Bruton's kinase inhibitor used in chronic myeloid leukaemia where prevalence of pulmonary hypertension $(\mathrm{PAH})$ is 5\%), cyclophosphomide and other alkylating agents [106, 107]. Echocardiography is preferred as the initial imaging modality with repeated assessment every 3-6 months recommended in patients receiving PAHassociated therapy $[10 \bullet \bullet]$.

\section{Cardiac masses}

Whilst echocardiography is generally the initial modality to detect cardiac masses (and is best for small, rapidly-moving masses including valve vegetations), assessing for tissue invasion and for differentiating mass aetiology generally requires advanced imaging techniques. CT has the spatial resolution to accurately determine the location, size and relationship of the mass to tissue planes, but CMR plays a key role [108, 109] in helping evaluate between different types of mass due to its inherent tissue characterisation sequences. After locating a mass on dark and bright blood sequences, T1- and T2-weighted imaging techniques, early and late gadolinium imaging and rest perfusion imaging can help determine the aetiology and potential resectability of the mass. Thrombi (common on indwelling venous catheters) can be easily detected using early gadolinium imaging, and malignant tumours are more likely to have heterogeneous signal intensity, cross tissue planes, and enhance on LGE and rest perfusion imaging [110]. Whilst a definitive malignant tissue diagnosis is rarely possible, key benign aetiologies can be detected (cysts, lipoma, thrombus). PET/CT imaging also has a role in differentiating benign from malignant tumours, and for detecting cardiac metastases [111] although careful patient preparation is key to obtaining diagnostic cardiac imaging.

\section{Light chain cardiac amyloidosis}

Patients with myeloma and other haematological malignancies rarely develop light chain amyloidosis, which may present with cardiac amyloidosis-an 


\section{Conclusions}

infiltrative cardiomyopathy, often presenting as heart failure with preserved ejection fraction (HFPEF). The diagnosis is often suspected from the characteristic echocardiographic appearances of left ventricular hypertrophy, preserved LV function, impaired RV function and profound apical sparing on strain maps [112]. Endomyocardial biopsy, whilst a definitive test if positive, carries inherent risks due to its invasive nature, meaning that a non-invasive diagnostic test is needed. CMR findings in cardiac amyloid are characteristic and can be used as prognostic markers, with left ventricular hypertrophy, abnormal gadolinium kinetics, subendocardial or transmural late gadolinium enhancement and significantly elevated myocardial T1 and ECV levels often detected in patients with cardiac involvement [113]. Nuclear bone scintigraphy techniques (using ${ }^{99 \mathrm{~m}} \mathrm{Tc}-$ PYP and ${ }^{99 \mathrm{~m}} \mathrm{Tc}$-DPD) can help differentiate light chain (AL) from transthyretin amyloidosis and a novel PET radiotracer (18F-florbetapir) [114] has recently been shown to be of potential value for detecting and quantifying AL amyloid in the heart.

Improved outcomes in oncology mean that it is increasingly important to prevent, detect and treat any early signs of treatment-related cardiotoxicity so patients can receive optimal cancer treatment but minimize subsequent cardiovascular morbidity and mortality. Advanced cardiac imaging techniques offer more sensitive and reproducible screening options than conventional 2D echocardiography or MUGA, and may provide novel insights into the underlying pathophysiology of CTRCD. The development of novel cancer treatments is currently rapid, and often cardiotoxicity is not detected until after initial phase 1 and 2 safety trials [115], with the causative mechanisms poorly understood. The need for accurate cardiac imaging biomarkers is therefore greater than ever, meaning that provision and access to CT, CMR and nuclear imaging will require expansion to match the growing demand from cardiooncology.

\section{Funding}

JCM and CM are directly and indirectly supported by the University College London Hospitals, National Institute for Health Research Biomedical Research Centre and Biomedical Research Unit at Barts Health NHS Trust. AS is supported by a doctoral research fellowship from the British Heart Foundation (FS/18/83/34025). $A B$ is supported by a doctoral research fellowship from the British Heart Foundation (FS/16/46/32187).

\section{Compliance with Ethical Standards}

\section{Conflict of Interest}

The authors declare that they have no conflict of interest.

Human and Animal Rights and Informed Consent

This article does not contain any studies with human or animal subjects performed by any of the authors. 


\section{Open Access}

This article is distributed under the terms of the Creative Commons Attribution 4.0 International License (http://creativecommons.org/licenses/by/4.0/), which permits unrestricted use, distribution, and reproduction in any medium, provided you give appropriate credit to the original author(s) and the source, provide a link to the Creative Commons license, and indicate if changes were made.

\section{References and Recommended Reading}

Papers of particular interest, published recently, have been highlighted as:

- Of importance

$\bullet \quad$ Of major importance

1. Heymach J, Krilov L, Alberg A, Baxter N, Chang SM, Corcoran RB, et al. Clinical Cancer Advances 2018: annual report on progress against cancer from the American Society of Clinical Oncology. J Clin Oncol. 2018;36(10):1020-44.

2. Cardinale D, Colombo A, Sandri MT, Lamantia G, Colombo N, Civelli M, et al. Prevention of high-dose chemotherapy-induced cardiotoxicity in high-risk patients by angiotensin-converting enzyme inhibition. Circulation. 2006;114(23):2474-81.

3. Pudil R. The future role of cardio-oncologists. Card Fail Rev. 2017;3(2):140-2.

4. Ghosh AK, Walker JM. Cardio-oncology - a new subspecialty with collaboration at its heart. Indian Heart J. 2017;69(4):556-62.

5. Albini A, Pennesi G, Donatelli F, Cammarota R, De Flora S, Noonan DM. Cardiotoxicity of anticancer drugs: the need for cardio-oncology and cardiooncological prevention. J Natl Cancer Inst. 2010;102(1):14-25.

6. Yoon GJ, Telli ML, Kao DP, Matsuda KY, Carlson RW, Witteles RM. Left ventricular dysfunction in patients receiving cardiotoxic cancer therapies are clinicians responding optimally? J Am Coll Cardiol. 2010;56(20):1644-50.

7. Savage P, Mahmoud S. Development and economic trends in cancer therapeutic drugs: a 5-year update 2010-2014. Br J Cancer. 2015;112(6):1037-41.

8. Kennedy JW, Sorensen SG, Ritchie JL, Folland ED, Hamilton GW. Radionuclide angiography for the evaluation of anthracycline therapy. Cancer Treat Rep. 1978;62(6):941-3.

9.•• Curigliano G, Cardinale D, Suter T, Plataniotis G, de Azambuja E, Sandri MT, et al. Cardiovascular toxicity induced by chemotherapy, targeted agents and radiotherapy: ESMO Clinical Practice Guidelines. Ann Oncol. 2012;23(Suppl 7):vii155-66

Guidelines/position statements from European Society of Medical Oncology (ESMO), European Society of Cardiology (ESC), American Society of Echocardiography (ASE), European Association of Cardiovascular Imaging (EACVI) and American Society of Clinical Oncology.
10.• Zamorano JL, Lancellotti P, Rodriguez Muñoz D, Aboyans V, Asteggiano R, Galderisi M, et al. 2016 ESC Position Paper on cancer treatments and cardiovascular toxicity developed under the auspices of the ESC Committee for Practice Guidelines: the task force for cancer treatments and cardiovascular toxicity of the European Society of Cardiology (ESC). Eur Heart J. 2016;37(36):2768-801

Guidelines/position statements from European Society of Medical Oncology (ESMO), European Society of Cardiology (ESC), American Society of Echocardiography (ASE), European Association of Cardiovascular Imaging (EACVI) and American Society of Clinical Oncology.

11.• Plana JC, Galderisi M, Barac A, Ewer MS, Ky B, ScherrerCrosbie M, et al. Expert consensus for multimodality imaging evaluation of adult patients during and after cancer therapy: a report from the American Society of Echocardiography and the European Association of Cardiovascular Imaging. Eur Heart J Cardiovasc Imaging. 2014;15(10):1063-93

Guidelines/position statements from European Society of Medical Oncology (ESMO), European Society of Cardiology (ESC), American Society of Echocardiography (ASE), European Association of Cardiovascular Imaging (EACVI) and American Society of Clinical Oncology.

12.• Armenian SH, Lacchetti C, Barac A, Carver J, Constine LS, Denduluri N, et al. Prevention and monitoring of cardiac dysfunction in survivors of adult cancers: American Society of Clinical Oncology Clinical Practice Guideline. J Clin Oncol. 2017;35(8):893-911

Guidelines/position statements from European Society of Medical Oncology (ESMO), European Society of Cardiology (ESC), American Society of Echocardiography (ASE), European Association of Cardiovascular Imaging (EACVI) and American Society of Clinical Oncology.

13.• Aapro M, Bernard-Marty C, Brain EGC, Batist G, Erdkamp F, Krzemieniecki K, et al. Anthracycline cardiotoxicity in the elderly cancer patient: a SIOG expert position paper. Ann Oncol. 2011;22(2):257-67 Guidelines/position statements from European Society of Medical Oncology (ESMO), European Society of Cardiology (ESC), American Society of Echocardiography (ASE), 
European Association of Cardiovascular Imaging (EACVI) and American Society of Clinical Oncology.

14. Denlinger CS, Sanft T, Baker KS, Broderick G, DemarkWahnefried W, Friedman DL, et al. Survivorship, Version 2.2018. J Natl Compr Cancer Netw. 2018;16(10):1216-47.

15. Gottdiener JS, Mathisen DJ, Borer JS, Bonow RO, Myers $\mathrm{CE}$, Barr LH, et al. Doxorubicin cardiotoxicity: assessment of late left ventricular dysfunction by radionuclide cineangiography. Ann Intern Med. 1981;94(4 pt 1):430-5.

16. Nousiainen T, Jantunen E, Vanninen E, Hartikainen J. Early decline in left ventricular ejection fraction predicts doxorubicin cardiotoxicity in lymphoma patients. Br J Cancer. 2002;86(11):1697-700.

17. Jensen BV, Skovsgaard T, Nielsen SL. Functional monitoring of anthracycline cardiotoxicity: a prospective, blinded, long-term observational study of outcome in 120 patients. Ann Oncol. 2002;13(5):699-709.

18. Chung R, Ghosh AK, Banerjee A. Cardiotoxicity: precision medicine with imprecise definitions. Open Heart. 2018 [cited 2019 May 6];5(2). Available from: https://www.ncbi.nlm.nih.gov/ pmc/articles/PMC6074618/.

19. Thavendiranathan P, Grant AD, Negishi T, Plana JC, Popović ZB, Marwick TH. Reproducibility of echocardiographic techniques for sequential assessment of left ventricular ejection fraction and volumes: application to patients undergoing cancer chemotherapy. J Am Coll Cardiol. 2013;61(1):77-84.

20. Katia Devorha Menacho Medina, Veronica Culotta, Anish N. Bhuva, Mark Westwood, Leon Menezes, James Moon, Charlotte Manisty. Precision of left ventricular ejection fraction measurement: comparison between 2D and 3D Echocardiography, Nuclear Imaging and Cardiovascular Magnetic Resonance. 22nd Annual SCMR Scientific Sessions; 2019.

21. Khouri MG, Douglas PS, Mackey JR, Martin M, Scott JM, Scherrer-Crosbie M, et al. Cancer therapy-induced cardiac toxicity in early breast cancer: addressing the unresolved issues. Circulation. 2012;126(23):2749-63.

22. Alherbish A, Becher H, Alemayehu W, Paterson DI, Butler CR, Anderson TJ, et al. Impact of contrast echocardiography on accurate discrimination of specific degree of left ventricular systolic dysfunction and comparison with cardiac magnetic resonance imaging. Echocardiography. 2018;35(11):1746-54.

23. Lang RM, Badano LP, Mor-Avi V, Afilalo J, Armstrong A, Ernande L, et al. Recommendations for cardiac chamber quantification by echocardiography in adults: an update from the American Society of Echocardiography and the European Association of Cardiovascular Imaging. J Am Soc Echocardiogr. 2015;28(1):139.e14.

24. Dorosz JL, Lezotte DC, Weitzenkamp DA, Allen LA, Salcedo EE. Performance of 3-dimensional echocardiography in measuring left ventricular volumes and ejection fraction: a systematic review and meta-analysis. J Am Coll Cardiol. 2012;59(20):1799-808.

25. Sugeng L, Mor-Avi V, Weinert L, Niel J, Ebner C, Steringer-Mascherbauer R, et al. Quantitative assessment of left ventricular size and function: side-by-side comparison of real-time three-dimensional echocardiography and computed tomography with magnetic resonance reference. Circulation. 2006;114(7):654-61.

26. Kühl HP, Schreckenberg M, Rulands D, Katoh M, Schäfer W, Schummers G, et al. High-resolution transthoracic real-time three-dimensional echocardiography: quantitation of cardiac volumes and function using semi-automatic border detection and comparison with cardiac magnetic resonance imaging. J Am Coll Cardiol. 2004;43(11):2083-90.

27. Jenkins C, Bricknell K, Hanekom L, Marwick TH. Reproducibility and accuracy of echocardiographic measurements of left ventricular parameters using real-time three-dimensional echocardiography. J Am Coll Cardiol. 2004;44(4):878-86.

28. Santoro C, Arpino G, Esposito R, Lembo M, Paciolla I, Cardalesi C, et al. 2D and 3D strain for detection of subclinical anthracycline cardiotoxicity in breast cancer patients: a balance with feasibility. Eur Heart J

Cardiovasc Imaging. 2017;18(8):930-6.

29. Walker J, Bhullar N, Fallah-Rad N, Lytwyn M, Golian $\mathrm{M}$, Fang T, et al. Role of three-dimensional echocardiography in breast cancer: comparison with twodimensional echocardiography, multiple-gated acquisition scans, and cardiac magnetic resonance imaging. J Clin Oncol. 2010;28(21):3429-36.

30. van Royen N, Jaffe CC, Krumholz HM, Johnson KM, Lynch PJ, Natale D, et al. Comparison and reproducibility of visual echocardiographic and quantitative radionuclide left ventricular ejection fractions. Am J Cardiol. 1996;77(10):843-50.

31. Huang H, Nijjar PS, Misialek JR, Blaes A, Derrico NP, Kazmirczak F, et al. Accuracy of left ventricular ejection fraction by contemporary multiple gated acquisition scanning in patients with cancer: comparison with cardiovascular magnetic resonance. J Cardiovasc Magn Reson. 2017;19(1):34.

32. Hacker M, Hoyer X, Kupzyk S, La Fougere C, Kois J, Stempfle H-U, et al. Clinical validation of the gated blood pool SPECT QBS processing software in congestive heart failure patients: correlation with MUGA, first-pass RNV and 2D-echocardiography. Int J Card Imaging. 2006;22(3-4):407-16.

33. Rajappan K, Livieratos L, Camici PG, Pennell DJ. Measurement of ventricular volumes and function: a comparison of gated PET and cardiovascular magnetic resonance. J Nucl Med. 2002;43(6):806-10.

34. Petersen SE, Aung N, Sanghvi MM, Zemrak F, Fung K, Paiva JM, et al. Reference ranges for cardiac structure and function using cardiovascular magnetic resonance (CMR) in Caucasians from the UK Biobank population cohort. J Cardiovasc Magn Reson. 2017 [cited 2018 
Nov 21];19. Available from: https://www.ncbi.nlm. nih.gov/pmc/articles/PMC5304550/.

35. Moody WE, Edwards NC, Chue CD, Taylor RJ, Ferro CJ, Townend JN, et al. Variability in cardiac MR measurement of left ventricular ejection fraction, volumes and mass in healthy adults: defining a significant change at 1 year. Br J Radiol. 2015;88(1049):20140831.

36. Grothues F, Smith GC, Moon JCC, Bellenger NG, Collins P, Klein HU, et al. Comparison of interstudy reproducibility of cardiovascular magnetic resonance with two-dimensional echocardiography in normal subjects and in patients with heart failure or left ventricular hypertrophy. Am J Cardiol. 2002;90(1):29-34.

37. Drafts BC, Twomley KM, D'Agostino R, Lawrence J, Avis N, Ellis LR, et al. Low to moderate dose anthracycline-based chemotherapy is associated with early noninvasive imaging evidence of subclinical cardiovascular disease. JACC Cardiovasc Imaging. 2013;6(8):877-85.

38. Gulati G, Heck SL, Ree AH, Hoffmann P, SchulzMenger J, Fagerland MW, et al. Prevention of cardiac dysfunction during adjuvant breast cancer therapy (PRADA): a 2 × 2 factorial, randomized, placebo-controlled, double-blind clinical trial of candesartan and metoprolol. Eur Heart J. 2016;37(21):1671-80.

39. Pituskin E, Mackey JR, Koshman S, Jassal D, Pitz M, Haykowsky MJ, et al. Multidisciplinary approach to novel therapies in cardio-oncology research (MANTICORE 101-Breast): a randomized trial for the prevention of trastuzumab-associated cardiotoxicity. J Clin Oncol. 2017;35(8):870-7.

40. Neilan TG, Coelho-Filho OR, Pena-Herrera D, Shah RV, Jerosch-Herold M, Francis SA, et al. Left ventricular mass in patients with a cardiomyopathy after treatment with anthracyclines. Am J Cardiol.

2012;110(11):1679-86.

41. Menacho K, Ramirez S, Segura P, Nordin S, AbdelGadir A, Illatopa V, et al. INCA (Peru) study: impact of non-invasive cardiac magnetic resonance assessment in the developing world. J Am Heart Assoc [Internet]. 2018 [cited 2019 Apr 25];7(17). Available from: https://www.ncbi.nlm.nih.gov/pmc/articles/ PMC6201420/.

42. Felker GM, Thompson RE, Hare JM, Hruban RH, Clemetson DE, Howard DL, et al. Underlying causes and long-term survival in patients with initially unexplained cardiomyopathy. N Engl J Med.

2000;342(15):1077-84.

43. Cardinale D, Colombo A, Lamantia G, Colombo N, Civelli M, De Giacomi G, et al. Anthracycline-induced cardiomyopathy: clinical relevance and response to pharmacologic therapy. J Am Coll Cardiol. 2010;55(3):213-20.

44. Cardinale D, Colombo A, Bacchiani G, Tedeschi I, Meroni $\mathrm{CA}$, Veglia F, et al. Early detection of anthracycline cardiotoxicity and improvement with heart failure therapy. Circulation. 2015;131(22):1981-8.

45. Ewer MS, Ali MK, Mackay B, Wallace S, Valdivieso M, Legha SS, et al. A comparison of cardiac biopsy grades and ejection fraction estimations in patients receiving Adriamycin. J Clin Oncol. 1984;2(2):112-7.

46. Neilan TG, Jassal DS, Perez-Sanz TM, Raher MJ, Pradhan AD, Buys ES, et al. Tissue Doppler imaging predicts left ventricular dysfunction and mortality in a murine model of cardiac injury. Eur Heart J. 2006;27(15):1868-75.

47. Stoddard MF, Seeger J, Liddell NE, Hadley TJ, Sullivan DM, Kupersmith J. Prolongation of isovolumetric relaxation time as assessed by Doppler echocardiography predicts doxorubicin-induced systolic dysfunction in humans. J Am Coll Cardiol. 1992;20(1):62-9.

48. Dorup I, Levitt G, Sullivan I, Sorensen K. Prospective longitudinal assessment of late anthracycline cardiotoxicity after childhood cancer: the role of diastolic function. Heart. 2004;90(10):1214-6.

49. Kirkham AA, Virani SA, Campbell KL. The utility of cardiac stress testing for detection of cardiovascular disease in breast cancer survivors: a systematic review. Int J Women's Health. 2015;7:127-40.

50. Jarfelt M, Kujacic V, Holmgren D, Bjarnason R, Lannering B. Exercise echocardiography reveals subclinical cardiac dysfunction in young adult survivors of childhood acute lymphoblastic leukemia. Pediatr Blood Cancer. 2007;49(6):835-40.

51. Civelli M, Cardinale D, Martinoni A, Lamantia G, Colombo N, Colombo A, et al. Early reduction in left ventricular contractile reserve detected by dobutamine stress echo predicts high-dose chemotherapy-induced cardiac toxicity. Int J Cardiol. 2006;111(1):120-6.

52. Bountioukos M, Doorduijn JK, Roelandt JRTC, Vourvouri EC, Bax JJ, Schinkel AFL, et al. Repetitive dobutamine stress echocardiography for the prediction of anthracycline cardiotoxicity. Eur J Echocardiogr. 2003;4(4):300-5.

53. Thavendiranathan P, Poulin F, Lim K-D, Plana JC, Woo A, Marwick TH. Use of myocardial strain imaging by echocardiography for the early detection of cardiotoxicity in patients during and after cancer chemotherapy: a systematic review. J Am Coll Cardiol. 2014;63(25 Pt A):2751-68.

54. Poterucha JT, Kutty S, Lindquist RK, Li L, Eidem BW. Changes in left ventricular longitudinal strain with anthracycline chemotherapy in adolescents precede subsequent decreased left ventricular ejection fraction. J Am Soc Echocardiogr. 2012;25(7):733-40.

55. Stoodley PW, Richards DAB, Boyd A, Hui R, Harnett PR, Meikle SR, et al. Left ventricular systolic function in HER2/neu negative breast cancer patients treated with anthracycline chemotherapy: a comparative analysis of left ventricular ejection fraction and myocardial strain imaging over 12 months. Eur J Cancer. 2013;49(16):3396-403.

56. Anqi Y, Yu Z, Mingjun X, Xiaoli K, Mengmeng L, Fangfang L, et al. Use of echocardiography to monitor myocardial damage during anthracycline chemotherapy. Echocardiography. 2019;36(3):495-502.

57. Mornoș C, Petrescu L. Early detection of anthracyclinemediated cardiotoxicity: the value of considering both 
global longitudinal left ventricular strain and twist. Can J Physiol Pharmacol. 2013;91(8):601-7.

58. Sawaya H, Sebag IA, Plana JC, Januzzi JL, Ky B, Tan TC, et al. Assessment of echocardiography and biomarkers for the extended prediction of cardiotoxicity in patients treated with anthracyclines, taxanes, and trastuzumab. Circ Cardiovasc Imaging. 2012;5(5):596-603.

59. Fallah-Rad N, Walker JR, Wassef A, Lytwyn M, Bohonis $\mathrm{S}$, Fang $\mathrm{T}$, et al. The utility of cardiac biomarkers, tissue velocity and strain imaging, and cardiac magnetic resonance imaging in predicting early left ventricular dysfunction in patients with human epidermal growth factor receptor II-positive breast cancer treated with adjuvant trastuzumab therapy. J Am Coll Cardiol. 2011;57(22):2263-70.

60. Song F-Y, Shi J, Guo Y, Zhang C-J, Xu Y-C, Zhang Q-L, et al. Assessment of biventricular systolic strain derived from the two-dimensional and three-dimensional speckle tracking echocardiography in lymphoma patients after anthracycline therapy. Int J Card Imaging. 2017;33(6):857-68.

61. Zhang KW, Finkelman BS, Gulati G, Narayan HK, Upshaw J, Narayan V, et al. Abnormalities in 3dimensional left ventricular mechanics with anthracycline chemotherapy are associated with systolic and diastolic dysfunction. JACC Cardiovasc Imaging. 2018;11(8):1059-68.

62. Negishi T, Thavendiranathan P, Negishi K, Marwick TH. SUCCOUR investigators. Rationale and design of the strain surveillance of chemotherapy for improving cardiovascular outcomes: The SUCCOUR Trial. JACC Cardiovasc Imaging. 2018;11(8):1098-105.

63. Scatteia A, Baritussio A, Bucciarelli-Ducci C. Strain imaging using cardiac magnetic resonance. Heart Fail Rev. 2017;22(4):465-76.

64. Romano S, Judd RM, Kim RJ, Kim HW, Klem I, Heitner $\mathrm{J}$, et al. Association of feature-tracking cardiac magnetic resonance imaging left ventricular global longitudinal strain with all-cause mortality in patients with reduced left ventricular ejection fraction. Circulation. 2017; 135(23):2313-5.

65. Nakano S, Takahashi M, Kimura F, Senoo T, Saeki T, Ueda $S$, et al. Cardiac magnetic resonance imagingbased myocardial strain study for evaluation of cardiotoxicity in breast cancer patients treated with trastuzumab: a pilot study to evaluate the feasibility of the method. Cardiol J. 2016;23(3):270-80.

66. Lunning MA, Kutty S, Rome ET, Li L, Padiyath A, Loberiza F, et al. Cardiac magnetic resonance imaging for the assessment of the myocardium after doxorubicin-based chemotherapy. Am J Clin Oncol. 2015;38(4):377-81.

67. Ong G, Brezden-Masley C, Dhir V, Deva DP, Chan KKW, Chow C-M, et al. Myocardial strain imaging by cardiac magnetic resonance for detection of subclinical myocardial dysfunction in breast cancer patients receiving trastuzumab and chemotherapy. Int J Cardiol. 2018;261:228-33.
68. Moudgil R, Yeh ETH. Mechanisms of cardiotoxicity of cancer chemotherapeutic agents: cardiomyopathy and beyond. Can J Cardiol. 2016;32(7):863-870.e5.

69. Liu J, Mao W, Ding B, Liang C. ERKs/p53 signal transduction pathway is involved in doxorubicininduced apoptosis in $\mathrm{H} 9 \mathrm{c} 2$ cells and cardiomyocytes. Am J Physiol Heart Circ Physiol. 2008;295(5):H1956-65.

70. Neilan TG, Rothenberg ML, Amiri-Kordestani L, Sullivan RJ, Steingart RM, Gregory W, et al. Myocarditis associated with immune checkpoint inhibitors: an expert consensus on data gaps and a call to action. Oncologist. 2018;23(8):874-8.

71. Kosmas C, Kallistratos MS, Kopterides P, Syrios J, Skopelitis H, Mylonakis N, et al. Cardiotoxicity of fluoropyrimidines in different schedules of administration: a prospective study. J Cancer Res Clin Oncol. 2008; 134(1):75-82.

72. Asnani A. Cardiotoxicity of immunotherapy: incidence, diagnosis, and management. Curr Oncol Rep. 2018;20(6):44.

73. Liu E, Guha A, Jia K, Ayers AM, Boudoulas KD, Bertino $\mathrm{E}$, et al. Cardiogenic shock in a patient being treated with atezolizumab for metastatic non-small cell lung cancer. Lung Cancer. 2017;114:106-7.

74. Kwong RY, Chan AK, Brown KA, Chan CW, Reynolds HG, Tsang S, et al. Impact of unrecognized myocardial scar detected by cardiac magnetic resonance imaging on event-free survival in patients presenting with signs or symptoms of coronary artery disease. Circulation. 2006;113(23):2733-43.

75. O'Hanlon R, Grasso A, Roughton M, Moon JC, Clark S, Wage $\mathrm{R}$, et al. Prognostic significance of myocardial fibrosis in hypertrophic cardiomyopathy. J Am Coll Cardiol. 2010;56(11):867-74.

76. Lightfoot JC, D'Agostino RB, Hamilton CA, Jordan J, Torti FM, Kock ND, et al. Novel approach to early detection of doxorubicin cardiotoxicity by gadoliniumenhanced cardiovascular magnetic resonance imaging in an experimental model. Circ Cardiovasc Imaging. 2010;3(5):550-8.

77. Thavendiranathan P, Wintersperger BJ, Flamm SD, Marwick TH. Cardiac MRI in the assessment of cardiac injury and toxicity from cancer chemotherapy: a systematic review. Circ Cardiovasc Imaging. 2013;6(6):1080-91.

78. Maestrini V, Cheang MH, Kotwinski P, Rosmini S, Lloyd G, Kellman P, et al. Late anthracycline-related cardiotoxicity in low-risk breast cancer patients. J Am Coll Cardiol. 2017;69(20):2573-5.

79. Nakamori S, Dohi K, Ishida M, Goto Y, ImanakaYoshida K, Omori T, et al. Native T1 mapping and extracellular volume mapping for the assessment of diffuse myocardial fibrosis in dilated cardiomyopathy. JACC Cardiovasc Imaging. 2018;11(1):48-59.

80. Flett AS, Hayward MP, Ashworth MT, Hansen MS, Taylor AM, Elliott PM, et al. Equilibrium contrast cardiovascular magnetic resonance for the measurement of diffuse myocardial fibrosis: 
preliminary validation in humans. Circulation. 2010;122(2):138-44.

81. Jordan JH, Vasu S, Morgan TM, D'Agostino RB, Meléndez GC, Hamilton CA, et al. Anthracyclineassociated T1 mapping characteristics are elevated independent of the presence of cardiovascular comorbidities in cancer survivors. Circ Cardiovasc Imaging. 2016;9(8).

82. Neilan TG, Coelho-Filho OR, Shah RV, Feng JH, PenaHerrera D, Mandry D, et al. Myocardial extracellular volume by cardiac magnetic resonance imaging in patients treated with anthracycline-based chemotherapy. Am J Cardiol. 2013;111(5):717-22.

83. Kimball A, Patil S, Koczwara B, Raman KS, Perry $\mathrm{R}$, Grover S, et al. Late characterisation of cardiac effects following anthracycline and trastuzumab treatment in breast cancer patients. Int J Cardiol. 2018;261:159-61.

84. Galán-Arriola C, Lobo M, Vílchez-Tschischke JP, López GJ, de Molina-Iracheta A, Pérez-Martínez C, et al. Serial magnetic resonance imaging to identify early stages of anthracycline-induced cardiotoxicity. J Am Coll Cardiol. 2019;73(7):779-9.

Study in a large animal model showing that $\mathrm{T} 2$ mapping abnormalities provide the earliest marker of subtle myocardial damage following anthracycline treatment, with $\mathrm{T} 2$ relaxation times prolonged long before LV motion abnormalities are detected.

85. Mahmood SS, Fradley MG, Cohen JV, Nohria A, Reynolds KL, Heinzerling LM, et al. Myocarditis in patients treated with immune checkpoint inhibitors. J Am Coll Cardiol. 2018;71(16):175564.

86. Nensa F, Kloth J, Tezgah E, Poeppel TD, Heusch P, Goebel J, et al. Feasibility of FDG-PET in myocarditis: comparison to CMR using integrated PET/MRI. J Nucl Cardiol. 2018;25(3):785-94.

87. Borde C, Kand P, Basu S. Enhanced myocardial fluorodeoxyglucose uptake following Adriamycinbased therapy: evidence of early chemotherapeutic cardiotoxicity? World J Radiol. 2012;4(5):220-3.

88. Bauckneht M, Ferrarazzo G, Fiz F, Morbelli S, Sarocchi $\mathrm{M}$, Pastorino F, et al. Doxorubicin effect on myocardial metabolism as a prerequisite for subsequent development of cardiac toxicity: a translational 18F-FDG PET/ CT observation. J Nucl Med. 2017;58(10):1638-45.

89. Shakir DK, Rasul KI. Chemotherapy induced cardiomyopathy: pathogenesis, monitoring and management. J Clin Med Res. 2009;1(1):8-12.

90. Barthur A, Brezden-Masley C, Connelly KA, Dhir V, Chan KKW, Haq R, et al. Longitudinal assessment of right ventricular structure and function by cardiovascular magnetic resonance in breast cancer patients treated with trastuzumab: a prospective observational study. J Cardiovasc Magn Reson. 2017;19(1):44.

91. Tadic M, Cuspidi C, Hering D, Venneri L, Danylenko $\mathrm{O}$. The influence of chemotherapy on the right ventricle: did we forget something? Clin Cardiol. 2017;40(7):437-43.
92. Andrejak M, Tribouilloy C. Drug-induced valvular heart disease: an update. Arch Cardiovasc Dis. 2013;106(5):333-9.

93. Murbraech K, Wethal T, Smeland KB, Holte H, Loge JH, Holte $\mathrm{E}$, et al. Valvular dysfunction in lymphoma survivors treated with autologous stem cell transplantation: a national cross-sectional study. JACC Cardiovasc Imaging. 2016;9(3):230-9.

94. Das D, Asher A, Ghosh AK. Cancer and coronary artery disease: common associations, diagnosis and management challenges. Curr Treat Options in Oncol. 2019;20(6):46.

95. Danad I, Szymonifka J, Twisk JWR, Norgaard BL, Zarins CK, Knaapen P, et al. Diagnostic performance of cardiac imaging methods to diagnose ischaemia-causing coronary artery disease when directly compared with fractional flow reserve as a reference standard: a metaanalysis. Eur Heart J. 2017;38(13):991-8.

96. Engblom H, Xue H, Akil S, Carlsson M, Hindorf C, Oddstig J, et al. Fully quantitative cardiovascular magnetic resonance myocardial perfusion ready for clinical use: a comparison between cardiovascular magnetic resonance imaging and positron emission tomography. J Cardiovasc Magn Reson. 2017;19:78.

97. Gujral DM, Lloyd G, Bhattacharyya S. Radiationinduced valvular heart disease. Heart. 2016;102(4):269-76.

98. Donnellan E, Masri A, Johnston DR, Pettersson GB, Rodriguez LL, Popovic ZB, et al. Long-term outcomes of patients with mediastinal radiation-associated severe aortic stenosis and subsequent surgical aortic valve replacement: a matched cohort study. J Am Heart Assoc. 2017;6(5).

99. Donnellan E, Krishnaswamy A, Hutt-Centeno E, Johnston DR, Aguilera J, Kapadia SR, et al. Outcomes of patients with mediastinal radiation-associated severe aortic stenosis undergoing transcatheter aortic valve replacement. Circulation. 2018;138(16):1752-4.

100. Dursun M, Yilmaz S, Yilmaz E, Yılmaz R, Onur I, Oflaz $\mathrm{H}$, et al. The utility of cardiac MRI in diagnosis of infective endocarditis: preliminary results. Diagn Interv Radiol. 2015;21(1):28-33.

101. Koo HJ, Yang DH, Kang J-W, Lee JY, Kim D-H, Song J-M, et al. Demonstration of infective endocarditis by cardiac CT and transoesophageal echocardiography: comparison with intraoperative findings. Eur Heart J Cardiovasc Imaging. 2018;19(2):199-207.

102. Swart LE, Gomes A, Scholtens AM, Sinha B, Tanis W, Lam MGEH, et al. Improving the diagnostic performance of 18 F-fluorodeoxyglucose positron-emission tomography/computed tomography in prosthetic heart valve endocarditis. Circulation. 2018;138(14):1412-27.

103. Nishimura RA, Otto CM, Bonow RO, Carabello BA, Erwin JP, Guyton RA, et al. 2014 AHA/ACC guideline for the management of patients with valvular heart disease: executive summary: a report of the American College of Cardiology/ 
American Heart Association Task Force on Practice Guidelines. J Am Coll Cardiol. 2014;63(22):2438-88.

104. Ghosh AK, Crake T, Manisty C, Westwood M. Pericardial disease in cancer patients. Curr Treat Options Cardiovasc Med. 2018;20(7):60.

105. Bogaert J, Francone M. Cardiovascular magnetic resonance in pericardial diseases. J Cardiovasc Magn Reson. 2009;11:14.

106. Lee S-E, Kim S-H, Choi S-Y, Park J-E, Jeon H-R, Jang EJ, et al. Six-year follow-up of dasatinib-related pulmonary arterial hypertension (PAH) for chronic myeloid leukemia in single center. Blood. 2013;122(21):4017.

107. Ranchoux B, Günther S, Quarck R, Chaumais M-C, Dorfmüller P, Antigny F, et al. Chemotherapyinduced pulmonary hypertension: role of alkylating agents. Am J Pathol. 2015;185(2):356-71.

108. Fussen S, De Boeck BWL, Zellweger MJ, Bremerich J, Goetschalckx K, Zuber M, et al. Cardiovascular magnetic resonance imaging for diagnosis and clinical management of suspected cardiac masses and tumours. Eur Heart J. 2011;32(12):1551-60.

109. Motwani M, Kidambi A, Herzog BA, Uddin A, Greenwood JP, Plein S. MR imaging of cardiac tumors and masses: a review of methods and clinical applications. Radiology. 2013;268(1):26-43.

110. Pazos-López P, Pozo E, Siqueira ME, García-Lunar I, Cham M, Jacobi A, et al. Value of CMR for the differential diagnosis of cardiac masses. JACC Cardiovasc Imaging. 2014;7(9):896-905.

111. Rahbar K, Seifarth H, Schäfers M, Stegger L, Hoffmeier A, Spieker T, et al. Differentiation of malignant and benign cardiac tumors using 18F-FDG PET/CT. J Nucl Med. 2012;53(6):856-63.

112. Cacciapuoti F. The role of echocardiography in the non-invasive diagnosis of cardiac amyloidosis. J Echocardiogr. 2015;13(3):84-9.

113. Banypersad SM, Fontana M, Maestrini V, Sado DM, Captur G, Petrie A, et al. T1 mapping and survival in systemic light-chain amyloidosis. Eur Heart J. 2015;36(4):244-51.

114. Manwani R, Page J, Lane T, Burniston M, Skillen A, Lachmann HJ, et al. A pilot study demonstrating cardiac uptake with 18F-florbetapir PET in AL amyloidosis patients with cardiac involvement. Amyloid. 2018;25(4):247-52.

115. Zheng P-P, Li J, Kros JM. Breakthroughs in modern cancer therapy and elusive cardiotoxicity: critical research-practice gaps, challenges, and insights. Med Res Rev. 2018;38(1):325-76.

\section{Publisher's Note}

Springer Nature remains neutral with regard to jurisdictional claims in published maps and institutional affiliations. 